\title{
Subject strategies in part-whole transfer in free recall
}

\author{
NANCY SCHULZE and DAVID S. GORFEIN \\ New College, Sarasota, Florida 99578
}

\begin{abstract}
The effect of instructing subjects as to the nature of the relationship between the two lists of a part-to-whole transfer situation has been studied in a number of cases with conflicting results. Some studies have shown that instructions to the subject produce positive transfer, while in others only slight effects from instructions may be noted. Detailed analysis of subject output protocols in a part-to-whole experiment demonstrated that subjects who maintained their List 1 organization showed positive transfer to the whole list. Reanalysis of several studies in the literature supported the conclusion that subjects who maintain their organization will show positive transfer under these conditions. In a second experiment, it is demonstrated that subjects can be instructed to maintain their List 1 organization during List 2 and that such instruction enhances the amount of positive transfer obtained. The results are viewed as consistent with a hierarchical version of an organization theory for recall performance.
\end{abstract}

The organization theory of free recall is based to a large extent on Miller's (1956) unitization hypothesis, which holds that memory capacity is limited to approximately seven items or units. The way in which this memory limit is extended is by increasing the amount of information each unit contains, or, in other words, by recoding or reorganizing items into "chunks." Thus, in learning a list of unrelated words, the subjects must organize the words into what Tulving (1966) calls "higher order memory units" or "S-units." Improved performance over trials in free recall learning reflects the development of S-units and the subsequent increase in the size of these units.

One of the predictions made by organization theory is that the existence of inappropriate memory units should retard the learning of a free recall list. This is exactly what Tulving's (1966) part-whole transfer study was designed to test. He reasoned that if subjects learn part of a list and then attempt to learn the whole list, the memory units formed during part-list learning would be inappropriate for learning the whole list. Since organization theory assumes that there is a limit to the number of memory units retrievable on a given trial (and it seems likely that the subject would have developed the maximum number of retrievable units during first-list learning), the subject cannot merely form new units in addition to those already formed. Thus, to learn the whole list, the subject has two options: Add new words to the existing S-units, or abandon the part-list organization and form new S-units. Tulving (1966) states: "The first expedient; adding new words, may be applicable only on a limited scale if the items in the list have been

This study represents part of a senior thesis conducted by Ms. Schulze under the supervision of Dr. Gorfein. Reprints may be requested from David S. Gorfein, Department of Psychology, Adelphi University, Garden City, Long Island. New York 11530. selected randomly. The second alternative would probably require extra effort and time, offsetting any potential advantage of prior learning. Therefore under the conditions of free recall learning, prior learning of a part of the list may have very little facilitating effect on the learning of the whole list or perhaps even an inhibiting effect" (pp. 195-196).

Tulving had two groups of subjects learn identical whole lists of 36 unrelated words under standard free recall procedures. Prior to this, the experimental or part-learning group had been given eight free recall trials on half of the final list, whereas the control or no-learning group had received eight trials on an irrelevant list of the same length. The experimental group performed better than the control group on the early trials of whole list learning, but on the later trials the control subjects' performance was superior. The difference in the slopes of the two learning curves was highly significant. Tulving concluded from these results that inappropriate organization can retard the memorization of a free recall list, which is consistent with organization theory.

In 1972. Novinski demonstrated that if the subjects are aware of the relationship between the two lists, negative transfer is eliminated. The basic part-whole paradigm was employed, with the exception that half of the transfer subjects were informed of the relationship between lists while the remaining transfer subjects were not informed. There were no differences in first-list performance among the two experimental and the control groups. On second-list performance. the informed group is superior on all trials to the other two groups; comparison of the noninformed and control groups showed the typical negative transfer effect.

Informing the subjects of the relationship between the lists does not always eliminate negative transfer. 
Wood and Clark (1969) reported a study involving the same manipulation of awareness of list relationship that Novinski employed. In this study, the informed subjects performed better than the uninformed subjects, but both groups of transfer subjects were still negative relative to an informed control group.

Sternberg and Bower (1974) have failed to show positive transfer in an aware group as compared to a control group, although the aware condition in their experiment performed significantly better than an unaware negative transfer group. Their aware group shows the typical crossover effect that has been interpreted as negative transfer in the literature.

The occurrence of positive transfer in a group that has been alerted to the fact that the whole list contains all of the part list items can be interpreted in several ways. Novinski (1972) has interpreted positive transfer as indicating that her subjects in the alerted group tended to maintain their first-list organization, while the nonalerted subjects tried to form a new organization. This is in contrast to the position presented by Tulving (1966), who suggested that the negative transfer in a noninformed group was due to the subject's trying to accommodate the second list to the subjective organization they had formed for the first list. We may therefore extend Tulving's argument to suggest that it would seem reasonable to expect positive transfer in an alerted group since they would know the experimental conditions and would wisely abandon the inappropriate first-list organization. Slamecka. Moore, and Carey (1972) suggest that subjects may be uncertain about whether all the first-list itens occur in the second list, and uncertainty along with a desire to avoid errors may lead subjects to set a "high criterion" for responding which might result in withholding of responses which, in fact, would be correct if recalled. In their experiment, subjects encouraged to guess showed an absence of negative transfer. Their position would suggest that informing subjects might cause them to set their bias in a similar manner to the bias caused by instructions to guess. Anderson and Bower (1972) and Schwartz and Humphreys (1973) have argued for a list discrimination hypothesis. Basically, the suggestion is that subjects have difficulties associating two tags, a List 1 tag and a List 2 tag, with the same item and, therefore, when asked to recall may edit out List 1 responses. Instructions alerting the subject might serve the function of automatically causing all List 1 items to be marked with a List 2 tag.

Although a number of explanations exist for the occurrence of positive transfer in an alerted group in a part-to-whole transfer experiment, none of the proposed explanations seem to account for the fact that not all the studies in the literature show positive transfer. The present study was undertaken to see if a more refined analysis of the data in studies comparing alerted and nonalerted groups in part-to-whole transfer might further explicate the process. An impetus for the study was a series of pilot studies in our laboratory that indicated an absence of negative transfer, whether or not the subject was alerted to the relationship of the two lists.

\section{EXPERIMENT I}

\section{Method}

Subjects. Forty-eight New College students were paid for serving as subjects. All subjects were naive to part-whole transfer procedures.

Materials and Design. A!l lists were composed of unrelated AA Thorndike-Lorge words. All subjects learned the same 36-word final list. Prior to this. the control subjects received eight trials on an 18-word list which contained none of the final list items; the transfer subjects learned an 18-word list composed of half of the final-list items. At the start of second-list learning, half of the transfer subjects were informed about the relationship between the two lists; the remaining transfer subjects and the control subjects were told only that they were to learn another list of words which was twice as long as the first list.

Procedure. The words were presented by a Kodak Carousel projector at a rate of 1 word/sec. Recall was written, with $45 \mathrm{sec}$ for recall on each of the eight first-list trials and $90 \mathrm{sec}$ for recall on each of the eight second-list trials. Items were presented in different random orders for all trials. New and old items were randomized in second-list learning.

\section{Results}

There were no differences among the three conditions in recall performance on the first list.

There were no significant differences between the aware and unaware transfer subjects in second-list recall performance, $\mathrm{F}(1,22)=.36, \mathrm{MS}_{\mathrm{e}}=134.9$; consequently, the data from these two groups were pooled for the remaining analyses. Recall performance for the control group and the transfer group was then compared. The transfer group shows clear positive transfer relative to the control group. $F(1,46)=29.39, \mathrm{MS}_{\mathrm{e}}=126.65, \mathrm{p}<.001$. The interaction of Trials by Treatment is statistically significant, $\mathrm{F}(7,322)=9.52, \mathrm{MS}_{\mathrm{e}}=6.43, \mathrm{p}<.01$. However, this results from the magnitude of the difference on Trials 1 and $2(9.8$ and 6.8 items, respectively). Beyond Trial 2 , the learning curves are parallel, with Trial 8 performance for the control group having a mean of 27.6 items correctly recalled while the transfer group showed a mean of 31.4.

The recall protocols of subjects were analyzed on the basis of the suggestion of Novinski (1972) that subjects who maintain their first-list organization are more likely to show positive transfer as well as on our observations made during the data analysis. The recall protocols of the transfer subjects were separated into three groups according to the order in which they recalled the words in second-list learning. The procedure for separating the subjects into the three groups was first to label each correctly recalled word as new or old for each trial to determine if the number 


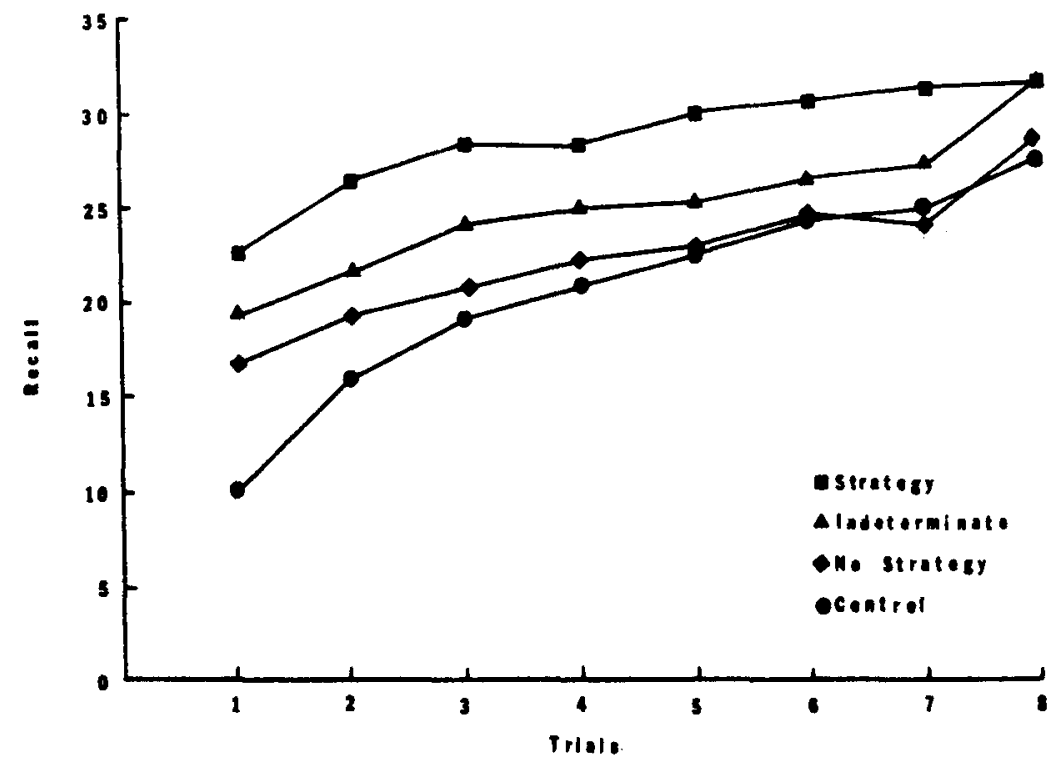

Figure 1. Second-list performance as a function of strategy group.

of new words in the first half of recall and the number of old words in the last half of recall exceeded that expected by chance. Subjects with five or more such trials were put into a "strategy group," subjects with three or fewer such trials were defined as "no strategy" subjects; the remaining subjects were put into the "indeterminate" group.

Figure 1 presents recall performance over trials for the three transfer groups and the control group. The strategy subjects $(\mathbf{N}=9)$ are superior to all the other groups; the indeterminate subjects $(N=9)$ are superior to the no-strategy subjects $(N=6)$ and the control group; the no-strategy group shows the typical negative part-whole transfer relative to the control group. An analysis of variance confirms these trends, showing significant effects of conditions $[F(3,44)=$ $\left.11.8, \mathrm{MS}_{\mathrm{e}}=116.59, \mathrm{p}<.001\right]$, trials $[\mathrm{F}(7,308)=$ $\left.163, \mathrm{MS}_{\mathrm{e}}=6.04, \mathrm{p}<.001\right]$, and the Conditions by Trials interaction $\left[\mathrm{F}(21,308)=4.35, \mathrm{MS}_{\mathrm{e}}=6.52\right.$, $\mathrm{p}<.01]$.

A direct examination was undertaken to see to what extent subjects in the three strategy groups maintained their first-list organization into second-list learning. A bidirectional cluster ratio (Gorfein, Blair, \& O'Neill. 1969) was employed to measure the stability of list organization from Trial 8 of the first list to Trial 1 of the second list. For the purposes of this measure, all second-list items, i.e., items that were not part of the first list, were ignored, and thus two first-list items that had been adjacent on Trial 8 of the first list were considered to be still adjacent, even when they were now separated by several new items, so long as they were not separated by any first-list recalls. This measurement of the constancy of the sequences produced a mean cluster ratio score for the strategy group of $.123, .096$ for the indeterminate group, and .047 for the no-strategy condition. An analysis of variance showed that this result was statistically significant, $F(2,21)=6.67, \mathrm{MS}_{\mathrm{e}}=.002$, $\mathrm{p}<.01$.

The question arises as to what determines whether subjects adopt consistent strategies or fail to do so. It can be hypothesized that these subjects are perhaps superior verbal learners or superior organizers. Therefore, an analysis was done on first-list learning comparing the performance of the three strategy groups. Consistent with the hypothesis that superior learners are more likely to adopt appropriate strategies, the strategy group recalled more than the other two groups and the no-strategy group performed worse than the other two groups. These differences are significant, $F(2,21)=4.47, \mathrm{MS}_{\mathrm{e}}=17.92$, $p<.05$. Further, analysis of the subjective organization score on the first list, i.e., the cluster score from Trial 7 to Trial 8 of first-list performance, showed the three groups falling in the same order, $\mathrm{F}(2,21)=7.5, \mathrm{MS}_{\mathrm{e}}=.003, \mathrm{p}<.01$.

\section{EXPERIMENT II}

The results of Experiment I are consistent with the suggestion made by Novinski (1972) that negative transfer in part-to-whole transfer is due to a failure to maintain first list organization. Subjects who do maintain their first-list organization tend to show positive transfer with respect to the appropriate control condition. The finding that subjects who adopt the appropriate strategy are superior on first-list learning raises questions as to the generality of the conclusion; perhaps only subjects who have high verbal abilities are successful in adopting this strategy. Therefore, a second experiment was undertaken to see if subjects could be instructed to adopt an appropriate strategy and thereby achieve positive transfer in this situation. Further, an alternative explanation for the data in Experiment I 


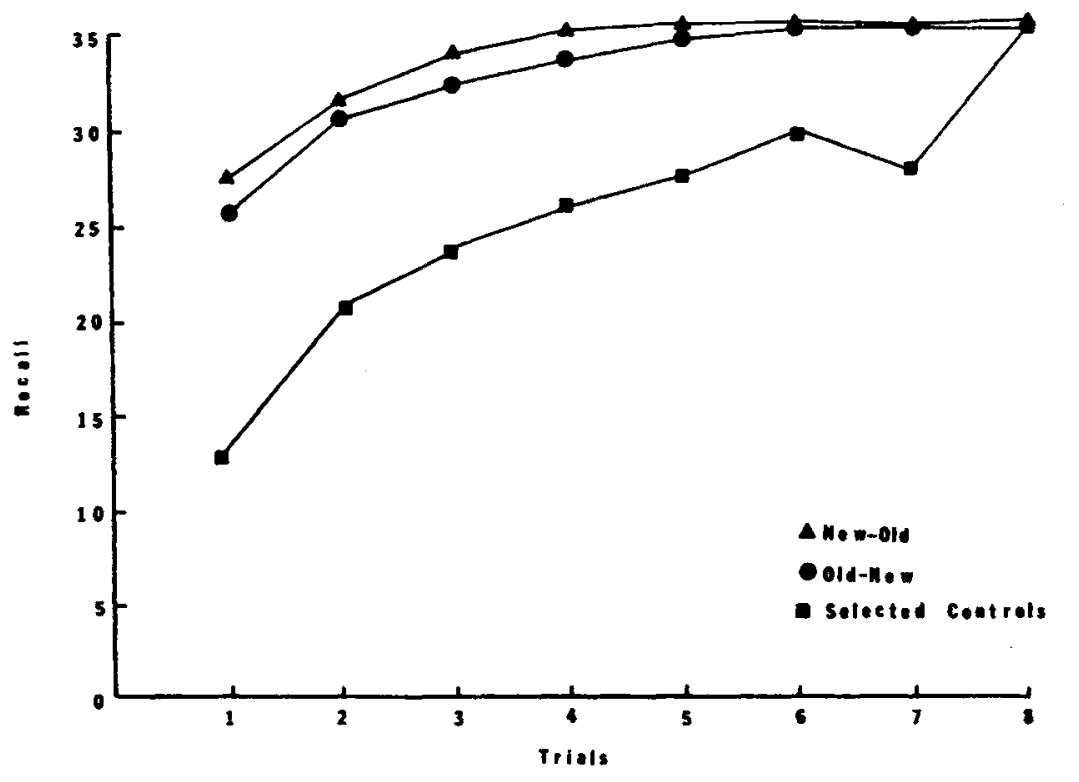

Figure 2. Performance as a function of instructed output order.

might be offered in terms of the nutput order adopted by the subjects. Perhaps outputting new words first helps the subjects recall, since it minimizes output interference on the weaker (List 2) words. Therefore, in Experiment II, half of the subjects were instructed. to recall new words first while half were instructed to recall the old (List 1) words first.

\section{Method}

Subjects. Twenty-four New College students served as subjects. Due to the small subject population at New College, these subjects regrettably were not naive to part-whole transfer procedures. None of these. however, had participated in Experiment I.

Materials and Design. The materials were identical to those employed in the transfer condition of Experiment I. All subjects received eight trials on each of two lists of words, with the first list being composed of half of the items in the second list. At the beginning of second-list learning, half of the subjects were instructed to recall first the oid items and then the new items, while the other half of the subjects were instructed to recall first the new items and then the old items.

Procedure. The procedure was identical to that followed in Experiment 1.

\section{Results}

There were no differences between the two conditions in first-list recall performance, $F(1,22)=$ $.446, \mathrm{MS}_{\mathrm{e}}=5.15$.

All subjects were able to follow the output order instructions as determined by a chi-square analysis of their List 2 trial protocols. Figure 2 shows mean recall performance for the two conditions in second-list learning. The group that was instructed to recall the new words first recalled slightly more words on every trial than did the group instructed to recall old words first, although the difference between the groups is not statistically reliable, $\mathrm{F}(1,22)=3.77, \mathrm{MS}_{\mathrm{e}}=$ $16.11, \mathrm{p}>.05$.

Since analysis of first-list learning indicated that these subjects were superior in their recall in List 1 to that of the control subjects in Experiment I, it was felt that it would be inappropriate to compare their performance to that of the control subjects in Experiment 1. However, a more conservative comparison was undertaken. It was possible to find four subjects in the Experiment I control group whose performance on first-list learning exceeded the mean performance for the subjects employed in Experiment II. Therefore, Figure 2 includes, for a basis of comparison, the mean performance for the selected subsample of control subjects from Experiment I. It can be seen that for all but the eighth serial position (where performances were very close to ceiling) there are large differences between the selected control condition and the two experimental groups.

For the results to be consistent with the maintenance of first-list organization hypothesis, it can be suggested that the subjects who are instructed in their ordered output should have a tendency to maintain their first-list organization into second-list learning. Therefore, we once again computed a bidirectional cluster score between Trial 8 of the first list and Trial 1 of the second list, utilizing the procedure described in Experiment $I$. The group instructed to recall new words first showed a mean $\mathrm{CR}$ for the old words of .172 , while those beginning the recall with the old words showed a mean of .198 . The two groups did not differ at a statistically significant level, $t(22)=.784, p>.05$. Since the two groups did not differ significantly, they were combined for comparison with the strategy subjects in Experiment 1 . The Experiment II subjects show significantly more carryover of first-list organization than the strategy subjects in Experiment $\mathrm{I}, \mathrm{t}(32)=2.38$, $\mathrm{p}<.05$. 


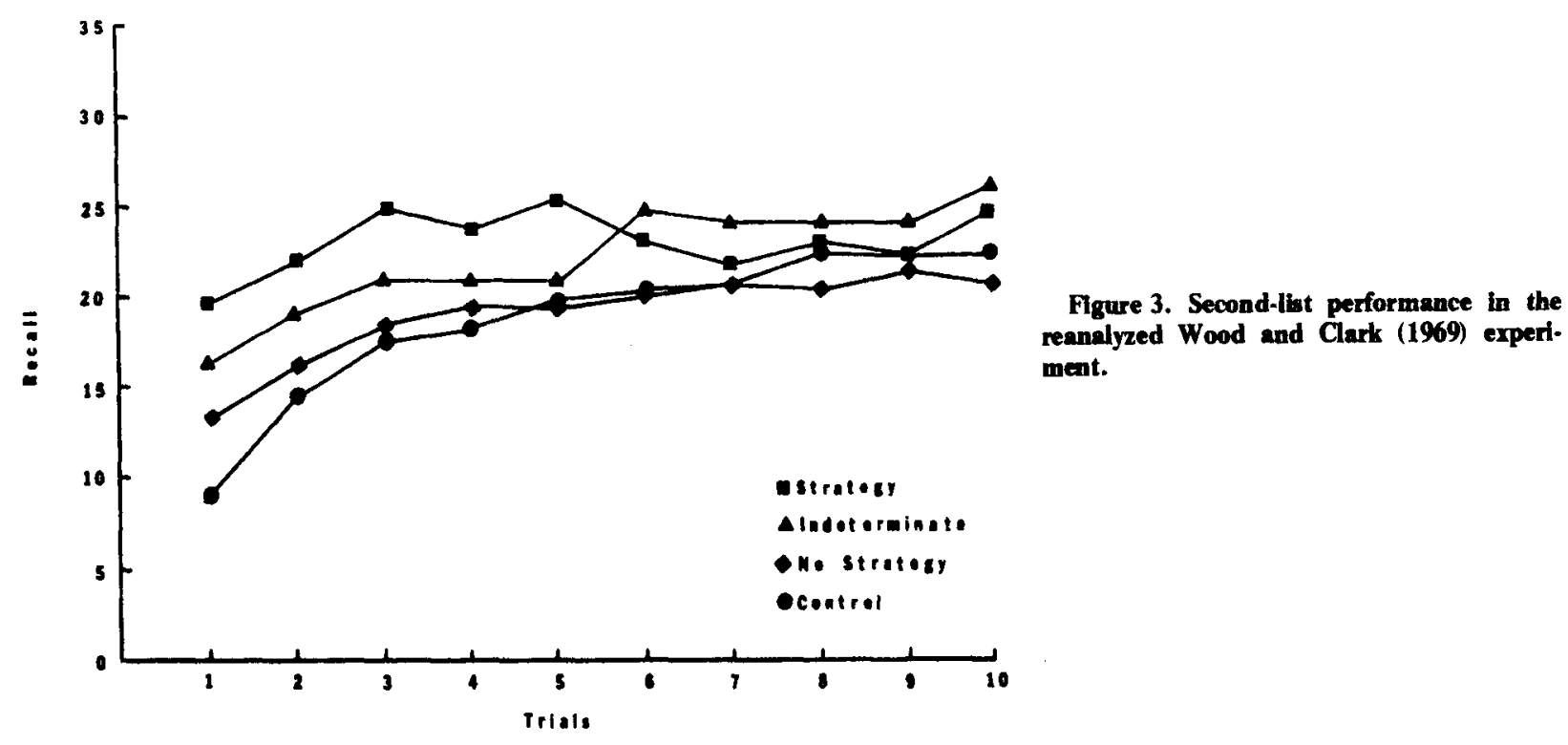

\section{DISCUSSION}

To this point, when discussing strategies in part-whole transfer, we have generally been referring to the strategy of recalling the new words before the old words in the output phase of learning, which does appear to improve recall performance. This is not the only possible strategy that could facilitate learning. The results of this study suggest that any manipulation that encourages the subject to maintain and add to his existing organization (rather than abandoning it and developing a new organization) will result in positive part-whole transfer. In this study, both sets of instructions tended to encourage the subjects to separate the old and new items. This would seem to facilitate the maintenance of the first-list organization and allow the subject to hierarchically organize the whole list, i.e., use list membership as an organizing cue. Thus both groups of subjects in this study were provided with efficient methods of organizing the material they were to learn, and both groups reached extremely high levels of performance in comparison with the subjects in the transfer condition of Experiment I, which employed the same materials. The group that recalled the new words first performed slightly better than the other group because, in recalling first the new words, the output interference (to which the new words are more susceptible than are the old words) is minimized, which gives a slight advantage to that group.

The results of these studies seem to suggest that the outcome of a part-whole transfer study may depend partially on the general verbal learning abilities of the subjects. The separation of transfer subjects according to the use or nonuse of the strategy of recalling first the new words and then the old words indicates that some subjects are capable of developing methods of learning or organizing that are sufficient to overcome the negative aspect of transferring from part of a list to the whole list. The fact that these subjects performed significantly better than the other subjects on both lists supports the idea that these subjects are better learners in general, as does the fact that these subjects had a higher level of organization and maintained more of their first-list organization than the other transfer subjects.

The discovery that the subjects who transferred positively to the whole list were also the subjects who were most successful at maintaining their part-list organization at first seems to completely contradict Tulving's argument that negative part-whole transfer is caused because part-list organization is inappropriate to whole-list learning and therefore retards performance on the whole list. The results seem to indicate, rather, as suggested by Novinski (1972), that the maintenance of the existing organization facilitates whole-list learning. Actually, the results show that Tulving was half right and half wrong in his reasoning concerning the outcome of transferring from part of a list to the whole list. He argues that abandoning the existing part-list organization and developing new organization to accommodate both the old and new words would offset any advantage there might be from prior learning of half of the whole list. The results of the organization analyses of the studies support the position that the performance of the subjects who abandon their first list organization is significantly inferior to that of the subject who maintains the first-list organization. Tulving seems to have been partly wrong, however, when he stated that it probably was not feasible for the subject to add new words to the existing organization. It may not be possible to add new words here and there to the 


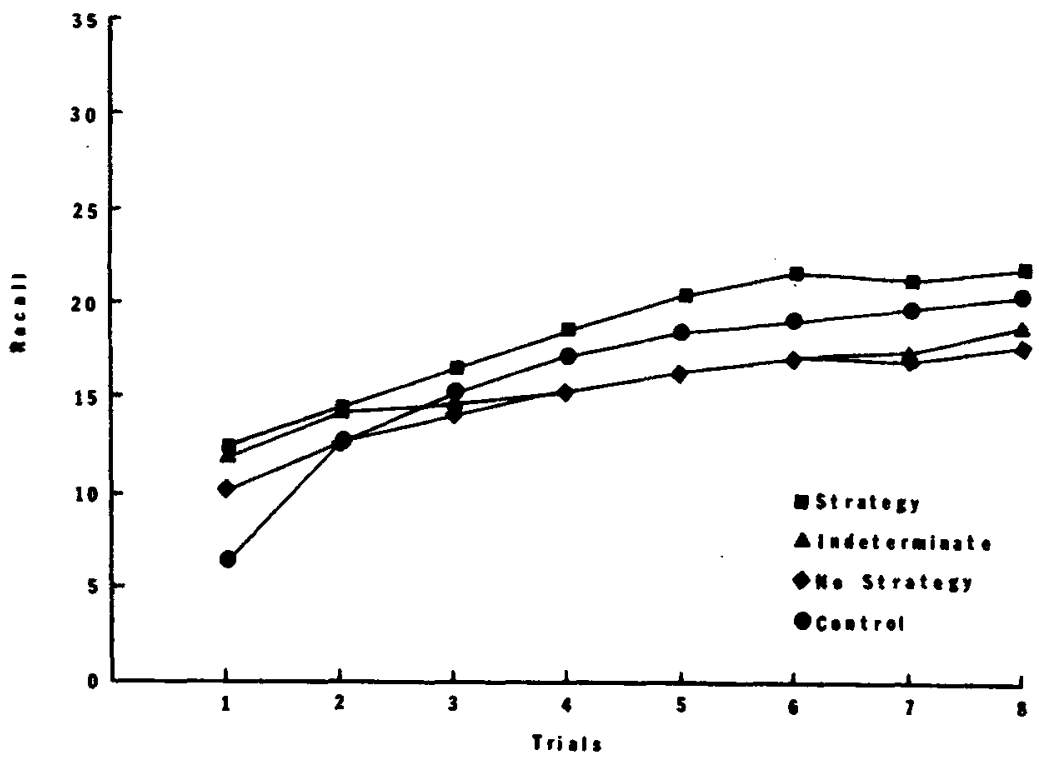

Figure 4. Second-list performance in the reanalyzed Stemberg and Bower (1974) experiment.

existing S-units, but it does seem that it should be possible to develop some sort of hierarchically structured organization of the whole list, with the two subsets of words (old and new) each as one unit or chunk. Within each chunk, there would be smaller chunks, the S-units. If this is the case, it would seem that any manipulation which would encourage the subject to treat the old and new words as distinct chunks would tend to produce positive transfer. Thus, informing subjects of the relationship between the lists probably causes more subjects to "discover" this effective method of organizing the items because it alerts them to the fact that there are two distinct sets of words in the list. It remains to be seen, however, if the hypothesis concerning the effects of instructions on subjects' recall strategies is sufficient to account for the contradictory findings of the studies in the literature.

Novinski (1972) reports that "the informed group of Ss recalled significantly more words on the second list than did the naive group and also showed a significantly greater tendency to organize the second list according to the categories of old and new words." To test the hypothesis with respect to the results of Wood and Clark (1969) and Sternberg and Bower (1974), we obtained ${ }^{1}$ the subject data protocols from the investigators in both of these studies. It is to be anticipated, then, since neither study obtained positive transfer, that very few of the subjects would show the pattern of recalling the items according to their list membership.

Figure 3 shows a reanalysis of the Wood and Clark data. Two of their instructed subjects showed the strategy, while none of their uninstructed group did so; 2 of the instructed subjects and 1 of the uninstructed are in the indeterminate category, while the remaining 12 instructed subjects and 15 of the 16 uninstructed subjects fell into the no-strategy group.
The results are consistent with the findings we obtained in Experiment $\mathbf{l}$ but fall short of statistical significance. An analysis among the three strategy groups shows $F(2.29)=2.63, \mathrm{MS}_{\mathrm{e}}=105.98$, $p>.05$.

Reanalysis of the Sternberg and Bower data shows that 5 of their 30 transfer subjects showed the strategy, 4 were in the indeterminate class, and the remaining 21 failed to show any signs of the strategy. Figure 4 shows the performance of these groups. Again, in this study, the performance in the strategy group is superior to that in the control condition over all trials, but the indeterminate group in this study fails to perform any better than the no-strategy condition, which shows the usial cross-over effect in relationship to the control group. Analysis among the three strategy conditions shows that the effects of the strategy falls just short of statistical significance, $\mathrm{F}(2,29)=3.16, \mathrm{MS}_{\mathrm{e}}=54.77, \mathrm{p}<.10$.

Overall, the experiments reported and the reanalysis of prior data seem to offer an explanation of some of the contradictory studies in the literature. In addition, they provide support for a hierarchical version of organization theory as an explanation of free recall performance.

The results of Experiment II also suggest that an efficient learning strategy can be induced by a verbal instruction, an important implication when one considers how many subjects in the "informed" conditions are unable to discover this strategy on their own.

\section{REFERENCES}

Anderson, J. R., \& Bower, G. H. Recognition and retrieval processes in free recall. Psychological Review, 1972, 79. 97-123. Gorfein, D. S., Blair, C., \& O'Neill, C. R. A reanalysis of "The generality of free recall: 1 . Subjective organization as an ability factor." Psychonomic Science, 1969, 17, 110. 
Miller. G. A. The magical number seven, plus or minus two: Some limits on our capacity for processing information Psychological Review, 1956, 63, 81-97.

Novinski. L. S. A reexamination of the part/whole effect in free recall. Journal of Verbal Learning and Verbal Behavior. $1972,11,229-233$.

SCHWARTz, R. M., \& Humphreys, M. S. List differentiation in part-whole free recall. American Journal of Psychology, 1973, 86, 79-88.

Slamecka, N. J., Moore, T., \& Carey, S. Part-to-whole transfer and its relation to organization theory. Joumal of Verbal Learning and Verbal Behavior, 1972, 11, 73-82.

STERnBerg, R. J., \& Bower, G. H. Transfer in part-whole and whole-part free recall: A comparative evaluation of theories. Journal of Verbal Learning and Verbal Behavior. 1974, 13, 1-26.
TulviNG, E. Subjective organization and the effects of repetition in multitrial free recall learning. Journal of Verbal Learning and Verbal Behavior, 1966, 5, 193-197.

Wood, G., \& ClaRk, D. Instructions, ordering, and previous practice in free recall learning. Psychonomic Science, 1969, $14,187-188$.

\section{NOTE}

1. The authors are indebted to Dr. Gordon Wood for making available the subject protocols from the Wood and Clark (1969) study and to Dr. Robert J. Sternberg for making available the subject protocols for the Sternberg and Bower (1974) study.

\section{(Received for publication August 4, 1975; revision received October 13,1975 .}

